MALAYA JOURNAL OF MATEMATIK

Malaya J. Mat. 09(02)(2021), 55-63.

http://doi.org/10.26637/mjm0902/007

\title{
On nearly Ricci recurrent manifolds
}

\author{
B. PRASAd ${ }^{1}$ AND R.P.S. YADAV*2 \\ ${ }^{1,2}$ Department of Mathematics, S.M.M.T.P.G. College, Ballia-277001, U.P., India.
}

Received 14 December 2020; Accepted 26 March 2021

\begin{abstract}
The object of the present paper is to introduce a new type of Ricci recurrent manifold called nearly Ricci recurrent manifold. Some geometric properties of nearly Ricci recurrent manifold have been studied. Finally we give an example of nearly Ricci recurrent manifold.
\end{abstract}

AMS Subject Classifications: 53C15 and 53C25.

Keywords: Nearly Ricci recurrent manifold, Constant scalar curvature tensor, Conformally flat manifold.

\section{Contents}

1 Introduction $\quad 55$

2 Preliminaries $\quad 57$

$3 \quad$ Existence of a $N\left\{R\left(R_{n}\right)\right\}(n \geq 2) \quad 57$

$4 \quad$ Nature of scalar curvature of a $N\left\{R\left(R_{n}\right)\right\} \quad 58$

$5 \quad$ Nature of the 1-forms $A$ and $B \quad 58$

$6 \quad N\left\{R\left(R_{n}\right)\right\}$ with constant scalar curvature $\quad \mathbf{5 8}$

7 Conformally flat $N\left\{R\left(R_{n}\right)\right\}$ with constant scalar curvature $\quad 59$

8 Necessary and sufficient condition for a $N\left\{R\left(R_{n}\right)\right\}$ to be a $(N R)_{n} \quad 60$

9 Example $\quad 61$

\section{Introduction}

Let $\left(M^{n}, g\right)$ be an n-dimensional Riemannian manifold with the matric $g$. A tensor field $T$ of type $(0, q)$ is said to recurrent [1] if the relation

$$
\left(D_{X} T\right)\left(Y_{1}, Y_{2}, \ldots, Y_{q}\right) T\left(Z_{1}, Z_{2}, \ldots, Z_{q}\right)-T\left(Y_{1}, Y_{2}, \ldots, Y_{q}\right)\left(D_{X} T\right)\left(Z_{1}, Z_{2}, \ldots, Z_{q}\right)=0
$$

holds on $\left(M^{n}, g\right)$. From definition it follows that if at a point $x \in M ; T(X) \neq 0$, then on some neighbourhood of $x$, there exits a unique 1-form $A$ satisfying

$$
\left(D_{X} T\right)\left(Y_{1}, Y_{2}, \ldots, Y_{q}\right)=A(X) T\left(Y_{1}, Y_{2}, \ldots, Y_{q}\right)
$$
*Corresponding author. Email address: bhagwatprasad2010@rediffmail.com (B. Prasad) and rana_2181@rediffmail.com (R.P.S.
Yadav) 


\section{B. Prasad and R.P.S. Yadav}

In 1952, Patterson [2] introduced a Ricci recurrent manifolds. According to him, a manifold $\left(M^{n}, g\right)$ of dimension $n$, was called Ricci recurrent if

$$
\left(D_{X} S\right)(Y, Z)=A(X) S(Y, Z)
$$

for some 1-form $A$. He denoted such a manifold by $R_{n}$. Ricci recurrent manifolds have been studied by several authors ( [3], [4], [1], [5] ) and many others. In a recent paper De, Guha and Kamilya [6] introduced the notion of generalized Ricci recurrent manifold as follows:

A non-flat Riemannian manifold $\left(M^{n}, g\right)(n>2)$ is called generalized Ricci recurrent if the Ricci tensor $S$ is non-zero and satisfies the condition:

$$
\left(D_{X} S\right)(Y, Z)=A(X) S(Y, Z)+B(X) g(Y, Z)
$$

where $A$ and $B$ non-zero 1 -forms. Such a manifold where denoted by them as $G R_{n}$. If the associated 1-form $B$ becomes zero, then the manifold $G R_{n}$ reduces to a Ricci recurrent manifold $R_{n}$. This justifies the name generalized Ricci recurrent manifold and the symbols $G R_{n}$ for it. Also in a paper De, and Guha [7] introduced a non flat Riemannian $\left(M^{n}, g\right)(n>2)$ called a generalized recurrent manifold if its curvature tensor $R(X, Y) Z$ of type $(1,3)$ satisfies the condition:

$$
\left(D_{U} R\right)(X, Y) Z=A(U) R(X, Y) Z+B(U)[g(Y, Z) X-g(X, Z) Y]
$$

where $A$ and $B$ are two non-zero 1-forms and $D$ denotes the operator of covariant differentiation with respect to metric tensor $g$. Such a manifold has been denoted by $G K_{n}$. If the associated 1-form $B$ becomes zero, then the manifold $G K_{n}$ reduces to recurrent manifold introduced by Ruse [8] and Waker [9] which was denoted by $K_{n}$. In recent papers Arslan etal [10], Shaikh and Patra [11], Mallick, De and De [12], Khairnar [Kh], Shaikh, Prakasha and Ahmad [14], Kumar, Singh and Chowdhary [15], Hui [16], Singh and Mayanglambam [17], Singh and Kishor [18] etc. explored various geometrical propertis by using generlaized recurrent and generlaized Ricci recurrent manifold on Riemannian manifolds, Lorentzian Trans-Sasakian manifolds, LP-Sasakian manifolds, $(k-\mu)$ contact metric manifolds.

Further the authors Prasad and Yadav [19] considered a non-flat Riemannian manifold $\left(M^{n}, g\right)(n>3)$ whose curvature tensor $\mathrm{R}$ satisfies the following condition:

$$
\left(D_{U} R\right)(X, Y) Z=[A(U)+B(U)] R(X, Y) Z+B(U)[g(Y, Z) X-g(X, Z) Y]
$$

where $A$ and $B$ are two non-zero 1-forms and $D$ has the meaning already mentioned. Such a manifold where called by them as nearly recurrent Riemannian manifold and denoted by $(N R)_{n}$.

The motivation of the above studies, we define a new type of non flat Riemannian manifold is called nearly Ricci recurrent manifolds if the Ricci tensor $S$ is non zero and satisfies the condition:

$$
\left(D_{X} S\right)(Y, Z)=[A(X)+B(X)] S(Y, Z)+B(X) g(Y, Z)
$$

where $A$ and $B$ non-zero 1-forms, $P$ and $Q$ be two vector fields such that

$$
A(X)=g(P, X), \quad B(X)=g(Q, X)
$$

Such a manifold shall be called as a nearly Ricci recurrent manifold and 1-forms $A$ and $B$ shall be called its associated 1-form and $n$ dimensional nearly Ricci recurrent manifold of this kind shall be denoted by $N\left\{R\left(R_{n}\right)\right\}$. The name nearly Ricci recurrent Riemannian manifold was chosen because if $B=0$ in (1.1) then the manifold reduces to a Ricci recurrent manifold which is very close to Ricci recurrent space. This justifies the name Nearly Ricci recurrent manifold for the manifold defined by (1.1) and the use of the symbol $N\left\{R\left(R_{n}\right)\right\}$ for it. 


\section{Nearly Ricci recurrent manifolds}

In this paper, after preliminaries, the existence of a $N\left\{R\left(R_{n}\right)\right\}$ is first established and then it proved that the scalar curvature of $N\left\{R\left(R_{n}\right)\right\}$ cannot be zero. In section 4, the necessary and sufficient condition for constant scalar curvature of $N\left\{R\left(R_{n}\right)\right\}$ is obtained. Here it is established if $A$ is closed then $B$ is also closed and conversely in section 5 . In section 6 , it is shown that if the scalar curvature is constant in $N\left\{R\left(R_{n}\right)\right\}$ then the eigen value of the Ricci tensor $S$ corresponding to the given eigen vector not exist. In section 7, it is proved that in Conformally flat $N\left\{R\left(R_{n}\right)\right\}$ with constant scalar curvature if the 1-form $A$ is closed then $R(X, Y) . S=0$ if and only if $\{A(X)+B(X)\} A(L Z)=\{A(Z)+B(Z)\} A(L X)$. In section 8 , a necessary and sufficient condition for $N\left\{R\left(R_{n}\right)\right\}$ to be a $(N R)_{n}$ is obtained. Finally the existence of nearly Ricci recurrent manifold $N\left\{R\left(R_{n}\right)\right\}$ is ensured by a non trivial example.

\section{Preliminaries}

Let $L$ denotes the symmetric endomorphism of the tangent space at each point of the manifold corresponding to the Ricci tensor $S$ that is $g(L X, Y)=S(X, Y)$ for every vector field $X, Y$. Therefore,

$$
g\left(\left(D_{X} L\right) Y, Z\right)=\left(D_{X} S\right)(Y, Z) .
$$

From (1.1), we have

$$
d r(X)=[A(X)+B(X)] r+n B(X) .
$$

\section{Existence of a $N\left\{R\left(R_{n}\right)\right\}(n \geq 2)$}

In this section, it show that there exist a Riemannian manifold $\left(M^{n}, g\right)(n \geq 2)$ whose Ricci tensor $S$ of type $(0,2)$ satisfies the condition

$$
\left(D_{X} S\right)(Y, Z)=[A(X)+B(X)] S(Y, Z)+B(X) g(Y, Z)
$$

and for which $\left(D_{X} S\right)(Y, Z) \neq A(X) S(Y, Z)$. For this we consider a Riemannian manifold $\left(M^{n}, g\right)$ which admits a linear connection $\bar{D}$ defined by

$$
\bar{D}_{X} Y=D_{X} Y+\frac{1}{2} B(X) L Y+\frac{1}{2} B(X) Y
$$

where $B$ is non zero 1 -form $L$ is a symmetric endomorphism of the tangent space at each point $\left(M^{n}, g\right)$ corresponding to the Ricci tensor $S$ defined by $g(L X, Y)=S(X, Y)$ and $L^{2} X=X$ and which satisfies the condition

$$
\left(\bar{D}_{X} S\right)(Y, Z)=A(X) S(Y, Z)
$$

If (3.2) holds, then

$$
\begin{aligned}
& X S(Y, Z)-S\left(\bar{D}_{X} Y, Z\right)-S\left(Y, \bar{D}_{X} Z\right)=A(X) S(Y, Z) \\
\Rightarrow & X S(Y, Z)-S\left(D_{X} Y+\frac{1}{2} B(X) L Y+\frac{1}{2} B(X) Y, Z\right)- \\
& S\left(Y, D_{X} Z+\frac{1}{2} B(X) L Z+\frac{1}{2} B(X) Z\right)=A(X) S(Y, Z)
\end{aligned}
$$

From this, we get

$$
\left(D_{X} S\right)(Y, Z)=[A(X)+B(X)] S(Y, Z)+B(X) g(Y, Z)
$$

The connection $D$ is not identical with $\bar{D}$. Hence $\left(D_{X} S\right)(Y, Z) \neq A(X) S(Y, Z)$. Thus a Riemannian manifold $\left(M^{n}, g\right)(n \geq 2)$ admits a linear connection $\bar{D}$ which satisfies (3.1) and (3.2) then the manifold is a $N\left\{R\left(R_{n}\right)\right\}$. 


\section{B. Prasad and R.P.S. Yadav}

\section{Nature of scalar curvature of a $N\left\{R\left(R_{n}\right)\right\}$}

From (2.2), we get if $r=0$, then $B=0$. Since the 1 -form $B$ cannot be zero.

Hence we can state the following theorem:

Theorem 4.1. The scalar curvature of a $N\left\{R\left(R_{n}\right)\right\}(n \geq 2)$ cannot be zero.

Now suppose that $N\left\{R\left(R_{n}\right)\right\}$ is of constant scalar curvature. Then from (2.2) it follows that

$$
[A(X)+B(X)] r+n B(X)=0
$$

Hence we have

$$
A(X)=-\left(1+\frac{n}{r}\right) B(X)
$$

Again if (4.1) holds, then from (2.2) we get $r=$ constant.

Hence we have the following theorem:

Theorem 4.2. $A N\left\{R\left(R_{n}\right)\right\}(n \geq 2)$ is of constant scalar curvature if and only if the condition (4.1) holds.

\section{Nature of the 1-forms $A$ and $B$}

We have

$$
d^{2} r(X, Y)=\frac{1}{2}[X d r(Y)-Y d r(X)-d r([X, Y])]
$$

Now in virtue of (2.2), we get from (5.1)

$$
\begin{aligned}
& \quad \frac{1}{2}[X\{(A(Y)+B(Y)) r+n B(Y)\}-Y\{(A(X)+B(X)) r+n B(X)\}-n B([X, Y])] \\
& \text { or } \operatorname{rd} A(X, Y)+(n+r) d B(X, Y)=0
\end{aligned}
$$

Since $B$ is closed then $r d A(X, Y)=0$. But $r \neq 0, A$ is closed.

Conversely if $A$ is closed then $B$ is closed.

Hence we have the following theorem:

Theorem 5.1. In a $N\left\{R\left(R_{n}\right)\right\}$ if $B$ is closed then $A$ is closed. Conversely if $A$ is closed then $B$ is closed, provided $r \neq 0$.

\section{6. $N\left\{R\left(R_{n}\right)\right\}$ with constant scalar curvature}

Let us suppose that the scalar curvature $r$ of a $N\left\{R\left(R_{n}\right)\right\}$ be constant. Now from (1.1), we have

$$
\begin{aligned}
\left(D_{X} S\right)(Y, Z)-\left(D_{Z} S\right)(Y, X)= & {[A(X)+B(X)] S(Y, Z)-[A(Z)+B(Z)] S(Y, X) } \\
& +B(X) g(Y, Z)-B(Z) g(Y, X)
\end{aligned}
$$

In view of (2.1), we have from (6.1)

$$
\begin{gathered}
g\left(\left(D_{X} L\right) Z, Y\right)-g\left(\left(D_{Z} L\right) X, Y\right)=[A(X)+B(X)] g(L Z, Y)-[A(Z)+B(Z)] g(L X, Y) \\
+B(X) g(Z, Y)-B(Z) g(X, Y) \\
\text { or }\left(D_{X} L\right) Z-\left(D_{Z} L\right) X=[A(X)+B(X)] L Z-[A(Z)+B(Z)] L X+B(X) Z-B(Z) X
\end{gathered}
$$

which on contraction gives

$$
d r(X)=2[A(X)+B(X)] r-2[A(L X)+B(L X)]+2(n-1) B(X) .
$$


From (2.2) and (6.2), we have

$$
\begin{gathered}
2[A(L X)+B(L X)]=[A(X)+B(X)] r+(n-2) B(X) \\
\text { or } B(X)=\frac{2}{r+n-2}[A(L X)+B(L X)]-\frac{r}{r+n-2} A(X)
\end{gathered}
$$

In view of (2.2) and (6.3), we get

$$
d r(X)=-\frac{2 r}{r+n-2} A(X)+\frac{2(r+n)}{r+n-2}[A(L X)+B(L X)] .
$$

Now if $r$ is constant then

$$
S(X, P)+S(X, Q)=\frac{1}{1+\frac{n}{r}} g(X, P)
$$

Hence we can state the following theorem:

Theorem 6.1. In a $N\left\{R\left(R_{n}\right)\right\}$, none of $P$ and $Q$ can be an eigen vector corresponding to any eigen values.

\section{Conformally flat $N\left\{R\left(R_{n}\right)\right\}$ with constant scalar curvature}

In Conformally flat $\left(M^{n}, g\right)$ it known [20]

$$
\left(D_{X} S\right)(Y, Z)-\left(D_{Z} S\right)(Y, X)=\frac{1}{2(n-1)}[d r(X) g(Y, Z)-d r(Z) g(X, Y)],
$$

From (2.2) and (7.1), we get

$$
\begin{aligned}
\left(D_{X} S\right)(Y, Z)-\left(D_{Z} S\right)(Y, X)= & \frac{1}{2(n-1)}[\{A(X)+B(X)\} \operatorname{rg}(Y, Z)+n B(X) g(Y, Z) \\
& -\{A(Z)+B(Z)\} r g(Y, X)-n B(Z) g(Y, X)] .
\end{aligned}
$$

Putting $Y=P$ in (7.2), we get

$$
\begin{gathered}
{[(A(X)+B(X)] A(L Z)-[(A(Z)+B(Z)] A(L X)+[B(X) A(Z)-B(Z) A(X)]=} \\
\frac{1}{2(n-1)}[\{A(X)+B(X)\} r A(Z)+n B(X) A(Z)-\{A(Z)+B(Z)\} r A(X)-n B(Z) A(X)] \\
\text { or } A(X) B(Z)-A(Z) B(X)=\frac{2(n-1)}{r-n+2}[\{A(X)+B(X)\} A(L Z) \\
-\{(A(Z)+B(Z)\} A(L X)]
\end{gathered}
$$

Now from (1.1),we get

$$
\begin{aligned}
\left(D_{U} D_{V} S\right)(Y, Z)= & {\left[\left(D_{U} A\right)(V)+A\left(D_{U} V\right)+\left(D_{U} B\right)(V)+B\left(D_{U} V\right)\right] S(Y, Z) } \\
& +[A(U)+B(U)][A(V)+B(V)] S(Y, Z)+ \\
& {\left[A(V) B(U)+B(U) B(V)+\left(D_{U} B\right)(V)+B\left(D_{U} V\right)\right] g(Y, Z) }
\end{aligned}
$$

From above, we have

$$
\begin{aligned}
& \left(D_{U} D_{V} S\right)(Y, Z)-\left(D_{V} D_{U} S\right)(Y, Z)-\left(D_{[U, V]} S\right)(Y, Z)= \\
& {\left[\left(D_{U} A\right)(V)-\left(D_{V} A\right)(U)+\left(D_{U} B\right)(V)-\left(D_{V} B\right)(U)\right] S(Y, Z)} \\
& {\left[\left(D_{U} B\right)(V)-\left(D_{V} B\right)(U)+A(V) B(U-A(U) B(V))\right] g(Y, Z)}
\end{aligned}
$$




\section{B. Prasad and R.P.S. Yadav}

which gives

$$
\begin{aligned}
(R(U, V) . S)(Y, Z)= & {[(d A(U, V)+d B(U, V)] S(Y, Z)+d B(U, V) g(Y, Z)} \\
& {[A(V) B(U)-A(U) B(V)] g(Y, Z) . }
\end{aligned}
$$

Suppose the 1-form $A$ is closed. Then in virtue of theorem (5.1) and (7.3) we get from (7.4)

$$
(R(U, V) . S)(Y, Z)=\frac{2(n-1)}{r-n+2}[\{A(X)+B(X)\} A(L Z)-\{A(Z)+B(Z)\} A(L X)]
$$

Hence we have the following theorem:

Theorem 7.1. In a Conformally flat $N\left\{R\left(R_{n}\right)\right\}$ with constant scalar curvature, $R(X, Y) . S=O$ if and only if $\{A(X)+B(X)\} A(L Z)=\{A(Z)+B(Z)\} A(L X)$.

\section{Necessary and sufficient condition for a $N\left\{R\left(R_{n}\right)\right\}$ to be a $(N R)_{n}$}

It is known that the Conformal curvature tensor ${ }^{\prime} C$ of type $(0,4)$ of a Riemannian manifold $\left(M^{n}, g\right)(n>3)$ is given by

$$
\begin{aligned}
{ }^{\prime} C(X, Y, Z, W)= & { }^{\prime} R(X, Y, Z, W)-\frac{1}{n-2}[S(Y, Z) g(X, W)-S(X, Z) g(Y, W) \\
& +S(X, W) g(Y, Z)-S(Y, W) g(X, Z)]- \\
& \frac{r}{(n-1)(n-2)}[g(Y, Z) g(X, W)-g(X, Z) g(Y, W)],
\end{aligned}
$$

where ${ }^{\prime} C(X, Y, Z, W)=g(C(X, Y) Z, W),{ }^{\prime} R(X, Y, Z, W)=g(R(X, Y) Z, W)$ and $C$ is the Conformal curvature tensor of type $(1,3)$. Now let $M^{n}$ be a nearly Ricci recurrent manifold $N\left\{R\left(R_{n}\right)\right\}$ specified by a non-zero1-form $B$.

Then in view of (1.1), (2.2) and (8.1), we get

$$
\begin{aligned}
& \left(D_{U}{ }^{\prime} C\right)(X, Y, Z, W)-[A(U)+B(U)]^{\prime} C(X, Y, Z, W)= \\
& \left(D_{U}{ }^{\prime} R\right)(X, Y, Z, W)-[A(U)+B(U)]^{\prime} R(X, Y, Z, W)- \\
& \frac{B(U)}{(n-1)}[g(Y, Z) g(X, W)-g(X, Z) g(Y, W)],
\end{aligned}
$$

Conversely if (8.2) holds, then putting $Y=Z=e_{j}$ in (8.2) where $\left\{e_{j}\right\}, j=1,2,3, \ldots, n$ is orthonormal basis of the tangent space at each point of the manifold and $l$ is summed for $l \leq j \leq n$, we get

$$
\begin{aligned}
& \left(D_{U} C\right)(X, W)-[A(U)+B(U)] C(X, W)= \\
& \left(D_{U} S\right)(X, W)-[A(U)+B(U)] S(X, W)-B(U) g(X, W)
\end{aligned}
$$

But in view of $C(X, W)=0$, we get from (8.3) that

$$
\left(D_{U} S\right)(X, W)-[A(U)+B(U)] S(X, W)-B(U) g(X, W) .
$$

From (8.2) and (8.3), we can state the following theorem:

Theorem 8.1. A necessary and sufficient condition that Riemannian manifold $M^{n}$ be a $N\left\{R\left(R_{n}\right)\right\}$ is that (8.2) holds.

In particular, if the $M^{n}$ Conformal to a flat space or if $n=3$ then $C=0$. In the first case it follows (8.2) that the $N\left\{R\left(R_{n}\right)\right\}$ is a $(N R)_{n}$. In the second case it follows that $N\left\{R\left(R_{3}\right)\right\}$ is a $(N R)_{3}$.

Thus we can state the following theorem:

Theorem 8.2. Every $N\left\{R\left(R_{n}\right)\right\}(n>3)$ is a $(N R)_{n}$ if it is Conformal to a flat space and every $N\left\{R\left(R_{3}\right)\right\}$ is $a(N R)_{3}$. 
Nearly Ricci recurrent manifolds

\section{Example}

Let us consider the 3-dimensional manifold $M=\left\{(x, y, z) \in R^{3}, z \neq 0\right\}$, where $(x, y, z)$ are standard coordinate of $R^{3}$.

We choose the vector fields

$$
e_{1}=e^{i y} \frac{\partial}{\partial x}, e_{2}=\frac{\partial}{\partial y}, e_{3}=e^{-i y} \frac{\partial}{\partial z}
$$

which is linearly independently at each point of $M$.

Let $g$ be the Riemannian metric denoted by

$$
g\left(e_{i}, e_{j}\right)= \begin{cases}1, & i=j \\ 0, & i \neq j\end{cases}
$$

Let $D$ be the Levi-Civita connection with respect to metric $g$. Then from equation (9.1), we have

$$
\left[e_{1}, e_{2}\right]=-i e_{1},\left[e_{1}, e_{3}\right]=0,\left[e_{2}, e_{3}\right]=-i e_{3} .
$$

The Riemannian connection $D$ of the metric $g$ is given by

$$
\begin{aligned}
2 g\left(D_{X} Y, Z\right)= & X g(Y, Z)+Y g(X, Z)-Z g(X, Y)-g(X,[Y, Z]) \\
& -g(Y,[X, Z])+g(Z,[X, Y]),
\end{aligned}
$$

which is known as Koszul's formula. Using (9.2) and (9.3) in (9.4), we get

$$
\begin{aligned}
& D_{e_{1}} e_{1}=i e_{2}, \quad D_{e_{1}} e_{2}=-i e_{1}, \quad D_{e_{1}} e_{3}=0 \\
& D_{e_{2}} e_{1}=0, \quad D_{e_{2}} e_{2}=0, \quad D_{e_{2}} e_{1}=0 \\
& D_{e_{3}} e_{1}=0, \quad D_{e_{3}} e_{2}=i e_{3}, \quad D_{e_{3}} e_{3}=-i e_{2} .
\end{aligned}
$$

The curvature tensor is given by

$$
R(X, Y) Z=D_{X} D_{Y} Z-D_{Y} D_{X} Z-D_{[X, Y]} Z
$$

Using (9.3) and (9.5) in (9.6), we get

$$
\begin{aligned}
& R\left(e_{1}, e_{2}\right) e_{1}=-e_{2}, \quad R\left(e_{1}, e_{2}\right) e_{2}=e_{1}, \quad R\left(e_{1}, e_{2}\right) e_{3}=0 \\
& R\left(e_{2}, e_{3}\right) e_{1}=0, \quad R\left(e_{2}, e_{3}\right) e_{2}=-e_{3}, \quad R\left(e_{2}, e_{3}\right) e_{3}=e_{2} \\
& R\left(e_{1}, e_{3}\right) e_{1}=e_{3}, \quad R\left(e_{1}, e_{3}\right) e_{2}=0 \quad R\left(e_{1}, e_{3}\right) e_{3}=-e_{1} \\
& R\left(e_{1}, e_{1}\right) e_{1}=R\left(e_{1}, e_{1}\right) e_{2}=R\left(e_{1}, e_{1}\right) e_{3}=0 \\
& R\left(e_{2}, e_{2}\right) e_{1}=R\left(e_{2}, e_{2}\right) e_{2}=R\left(e_{2}, e_{2}\right) e_{3}=0 \\
& R\left(e_{3}, e_{3}\right) e_{1}=R\left(e_{3}, e_{3}\right) e_{2}=R\left(e_{3}, e_{3}\right) e_{3}=0
\end{aligned}
$$

The Ricci tensor is given by

$$
S\left(e_{i}, e_{i}\right)=\sum_{i=1}^{3} g\left(R\left(e_{i}, X\right) Y, e_{i}\right)
$$

From (9.7) and (9.8), we get

$$
S\left(e_{1}, e_{1}\right)=0, \quad S\left(e_{2}, e_{2}\right)=2, \quad S\left(e_{3}, e_{3}\right)=0
$$

and the scalar curvature is $r=2$. 


\section{B. Prasad and R.P.S. Yadav}

Since $\left\{e_{1}, e_{2}, e_{3}\right\}$ forms a basis of Riemannian manifold any vector field $X, Y, Z \in \chi(M)$ can be written as

$$
X=a_{1} e_{1}+b_{1} e_{2}+c_{1} e_{3}, \quad Y=a_{2} e_{1}+b_{2} e_{2}+c_{2} e_{3},
$$

where $a_{j}, b_{j}, c_{j} \in \gtreqless+$ ( the set of all positive real numbers), $j=1,2,3$.

Hence

$$
\begin{gathered}
S(X, Y)=b_{1} b_{2} \\
g(X, Y)=a_{1} a_{2}+b_{1} b_{2}+c_{1} c_{2}
\end{gathered}
$$

By view of (9.10), we get

$$
\begin{gathered}
\left(D_{e_{j}} S\right)(X, Y)=D_{e_{j}} S(X, Y)-S\left(D_{e_{j}} X, Y\right)-S\left(X, D_{e_{j}} Y\right) \\
\left(D_{e_{1}} S\right)(X, Y)=-i\left(a_{1} b_{2}+a_{2} b_{1}\right) \\
\left(D_{e_{2}} S\right)(X, Y)=0 \\
\left(D_{e_{3}} S\right)(X, Y)=-i\left(b_{1} c_{2}+b_{2} c_{1}\right)
\end{gathered}
$$

Consequently, the manifold under consideration is neither Ricci symmetric nor Ricci recurrent. Let us now consider 1-form non vanishes

$$
\begin{aligned}
& A\left(e_{1}\right)=\frac{5 i\left(a_{1} b_{2}+a_{2} b_{1}\right)}{3 a_{1} a_{2}-b_{1} b_{2}+3 c_{1} c_{2}}, \quad B\left(e_{1}\right)=\frac{-3 i\left(a_{1} b_{2}+a_{2} b_{1}\right)}{3 a_{1} a_{2}-b_{1} b_{2}+3 c_{1} c_{2}} \\
& A\left(e_{2}\right)=0, \quad B\left(e_{2}\right)=0 \\
& A\left(e_{3}\right)=\frac{5 i\left(b_{1} c_{2}+b_{2} c_{1}\right)}{3 a_{1} a_{2}-b_{1} b_{2}+3 c_{1} c_{2}}, \quad B\left(e_{3}\right)=\frac{-3 i\left(b_{1} c_{2}+b_{2} c_{1}\right)}{3 a_{1} a_{2}-b_{1} b_{2}+3 c_{1} c_{2}}
\end{aligned}
$$

at any point $x \in M$. From (1.1), we have

$$
\left(D_{e_{j}} S\right)(X, Y)=\left[A\left(e_{j}\right)+B\left(e_{j}\right)\right] S(X, Y)+B\left(e_{j}\right) g(X, Y), \quad j=1,2,3 .
$$

It can be easily seen that the Riemannian manifold with 1-forms satisfies relation (9.13). Hence the manifold under consideration is a nearly Ricci recurrent manifold $\left(M^{3}, g\right)$, which is neither Ricci recurrent nor Ricci symmetric. Thus we have the following theorem:

Theorem 9.1. There exist a nearly Ricci recurrent manifold $\left(M^{3}, g\right)$, which is neither Ricci recurrent nor Ricci symmetric.

\section{References}

[1] W. Roter, On Conformally symmetric Ricci recurrent spaces, Colloquium Mathematicum., 31(1974), 8796.

[2] E.M. PaAtTerson, Some theorem on Ricci-recurrent space, J. London. Math. Soc., 27(1952), 287-295.

[3] M.C. Chaкi, Some theorem on recurrent and Ricci recurrent spaces, Rendicoti Seminario Math. Della universita Di Padova, 26(1956), 168-176.

[4] N. Prakash, A note on Ricci recurrent and recurrent spaces, Bull. Cal.Math. Society., 54(1962), 1-7.

[5] S.Yamaguchi and M. Matsumoto, On Ricci recurrent spaces, Tensor, N.S., 19(1968), 64-68. 
Nearly Ricci recurrent manifolds

[6] U.C. De, N. Guha And D. Kamilya, On generalized Ricci recurrent manifolds, Tensor (N.S.), 6(1995), 312-317.

[7] U.C. De AND N. GuHA, On generalized recurrent manifolds, National Academy of Math. India, 9(1991), 85-92.

[8] H.S.Ruse, A classification of K*-spaces, London Math. Soc., 53(1951), 212-229.

[9] A. G. WALKer, On Ruse's space of recurrent curvature,Proc. of London Math. Soc.,52(1950), 36-54.

[10] K. Arslan, U.C. De, C. Murathan and A. Yildiz, On generalized recurrent Riemannian manifolds, Acta Math. Hungar, 123(1-2)(2009), 27-39.

[11] A.A. SHAIKH AND A. PATRA, On a generalized class of recurrent manifolds, ARCHIVUM MATHEMATICUM (BRNO) Tomus, 46(2010), 71-78.

[12] S. Mallick, A. De and U.C. De, On Generalized Ricci Recurrent Manifolds with Applications To Relativity, Proc. Natl. Acad. Sci., India, Sect. A Phys. Sci., 83(2)(2013), 143-152.

[13] V.J. KHAIRNAR, On generalized recurrent and Ricci recurrent Lorentzian Trans Sasakian manifolds, IOSR J. Math. (IOSR-JM), 10(4) Ver.I(2014), 38-43.

[14] A.A. Shaikh, D.G. PraKasha AND H. Ahmad, On generalized $\phi$-recurrent LP-Sasakian manifolds, Journal of E. Math., Soc., 23(2015), 161-166.

[15] R. Kumar, J.P. Singh AND J. Chowdhury, On generalized Ricci recurrent LP-Sasakian manifolds, Journal of Mathematics and Computer science, 14(2015), 205-210.

[16] S.K. HuIL, On generalized $\phi$-recurrent generalized $(k-\mu)$ contact metric manifolds, arXiv Math., D.G., 11(2017), 1-10.

[17] J.P. Singh S.D. Mayanglambam, On extended generalized $\phi$-recurrent LP-Sasakian manifolds, Global Journal of Pure and Applied Mathematics, 13(2017), 5551-5563.

[18] A. Singh AND S. Kishor, Generalized recurrent and generalized Ricci recurrent Sasakian space forms, P.J. Math., 9(2)(2020), 866-873.

[19] B.PRASAD AND R.P.S. Yadav, On Nearly recurrent Riemannian manifolds, Communicated for publication.

[20] L.P. EIsenhert, Riemannian Geometry, Princeton University Press, Princetone, N.J. (1949).

This is an open access article distributed under the Creative Commons Attribution License, which permits unrestricted use, distribution, and reproduction in any medium, provided the original work is properly cited. 